\title{
Long-Term Chronic Diabetes Complications: the Cause, Cure and Prevention
}

\section{Owusu A Bempah*}

FV Supplement limited, United Kingdom

*Corresponding author: Owusu A Bempah, FV Supplement limited, United Kingdom, Tel: +44 (0)7914 120 175; E-mail: kb436@outlook.com

Received: 13 Jun, 2019 | Accepted: 24 Jun, 2019 | Published: 28 Jun, 2019

Citation: Bempah OA (2019) Long-Term Chronic Diabetes Complications: the Cause, Cure and Prevention. J Diab Res Ther 5(2): dx.doi. org/10.16966/2380-5544.143

Copyright: (c) 2019 Bempah OA. This is an open-access article distributed under the terms of the Creative Commons Attribution License, which permits unrestricted use, distribution, and reproduction in any medium, provided the original author and source are credited.

\begin{abstract}
Introduction: The flow-chart of diabetes progression, extended by empirical evidence of daily polyuria-induced Vitamin B deficiency in the blood plasma of diabetes patients, has provided a basis for a theory for the development, cure and prevention of long-term chronic diabetes complications.

Theory: The deficiency of Vitamins B1 and B3 causes vasoconstriction which impacts adversely on the exchange of nutrients, gases and particles between blood and tissue, resulting in tissue damage. With time, the accumulated tissue damage manifests as organ dysfunction-diabetes complications.
\end{abstract}

Hypothesis: Vitamin B supplementation to ensure full 24-hour vasodilatation in the microcirculation will stop tissue damage and allow cures of manifested symptoms, naturally.

Method: In on-going Open Trial beginning in 2008, tablets containing Vitamin B \{nicotinamide (15.0 mg); thiamine hydrochloride (12.5 mg); and Calcium-dpanthothenate $(11.0 \mathrm{mg})\}$, were ingested by patients with suspected diabetes complications in single/multiple organs.

Results: Over 200 cures reported included early-stage symptoms of complications in: the eye (retinopathy); kidney (swollen feet/ankles); skin sores (leg, gum, toe back, and head) and restored erectile functions. As prophylactic, the tablets have prevented manifestation of symptoms of complications for over 8 years. The new theory also explains the reported cures of diabetes complications accomplished with high doses of benfotiamine and thiamine.

Conclusion: So-called diabetes complications, like scurvy, are not a disease but manifestation of the effects of vitamin deficiency and are curable and preventable.

Keywords: Vitamin B deficiency model; Diabetes complications; Cure and prevention; Vitamin B supplementation

\section{Introduction}

"Diabetes" is a Greek word that means, "a siphon" [1]. This is a reference to the fact that people with the condition were observed to urinate copiously and more frequently. The urine of some patients was found (by observing ants feeding at dried up urine spots) to be honey-flavored, and of others, flavorless. The condition of passing honey-flavoured urine was described as Diabetes Mellitus (from Latin mel, honey), and when the urine was flavorless; Diabetes Insipidus (tasteless).

It is now known that the "honey flavor" comes from high blood plasma glucose concentration inadequately filtered by the kidney. The high blood plasma glucose concentration was caused by inadequate insulin production. The condition of extremely low or total lack of insulin production was described as Type 1 Diabetes Mellitus and moderately low insulin production, Type 2 . Flow-chart 1 shows our current understanding.
So, "copious and increased frequency in urination" - polyuria - is the event; "mellitus" and "insipidus" are merely descriptions of the flavor of the urine.

Blood plasma glucose and blood insulin levels are quantifiable and measurable. For this reason, diabetes mellitus has assumed centre-stage as the definition of diabetes. If one does not have high blood glucose level, one is declared a nondiabetic. Polyuria, though measurable, was seen as mere inconvenience.

Similarly, the parameters of all research efforts are defined in terms of hyperglycaemia. For example, the chronic, long-term diabetes complications that afflict diabetes patients are attributed to reaction products of plasma protein/glucose interaction. On this basis, persons afflicted with diabetes insipidus cannot suffer long-term chronic diabetes complications. This assumption is not justified. All efforts to find cure for, or prevention of, diabetes complications based on this glucose model-Glycation Theory, [2-4] have failed, so far. 


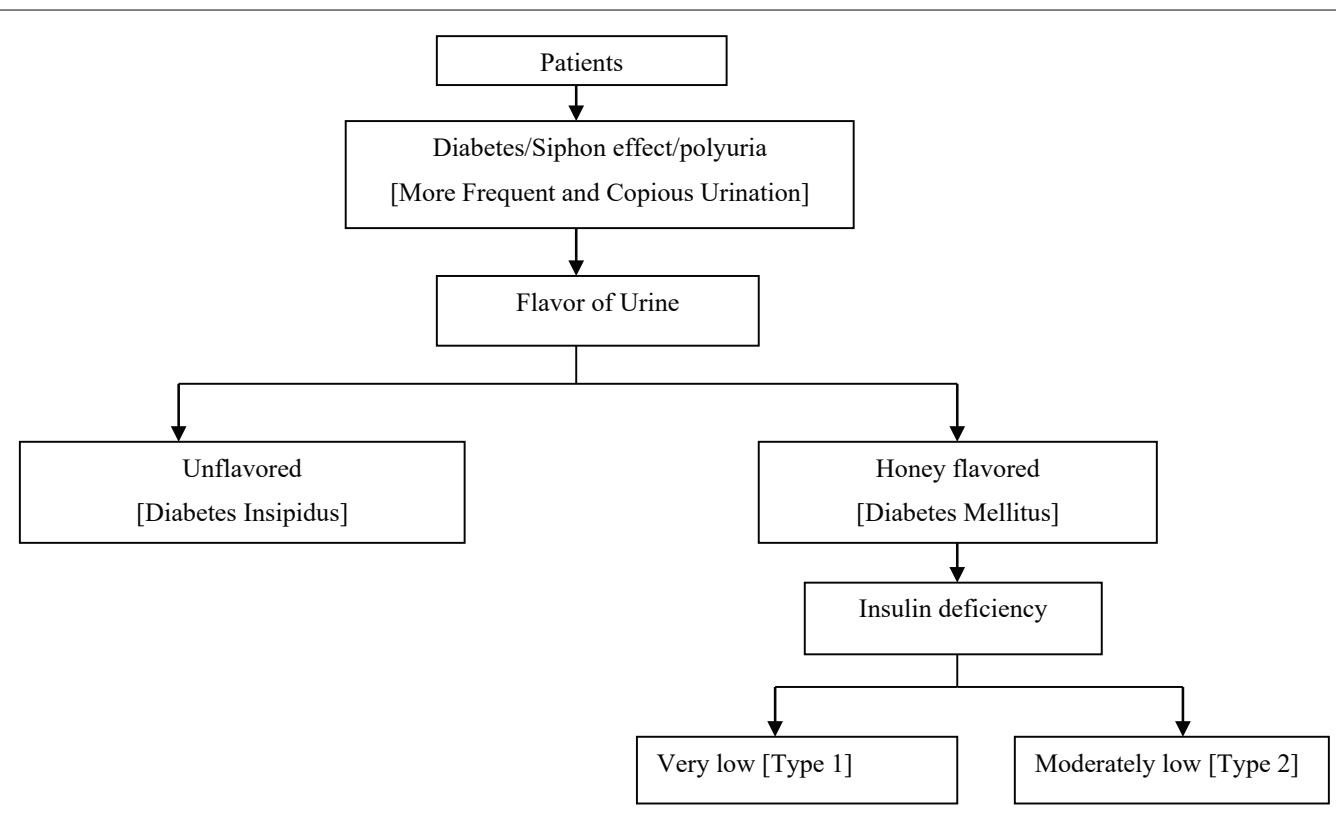

Flow-Chart 1: Flow-chart of the definition of Diabetes.

Table 1: The behavior of Thiamine in the blood plasma of Normal persons and Diabetes Patients in a 24-hour period.

\begin{tabular}{|l|c|c|c|}
\hline \multicolumn{1}{|c|}{ Category/Thiamine conc. nmol/I } & Type 1 (26) & Type 2 (48) & Healthy persons (20) \\
\hline Initial & 100 & 100 & 100 \\
\hline After 24 hours (Mean) SD & $15.4(9.6)$ & $16.3(11.5)$ & $64.1(12.1)$ \\
\hline Volume of urine & $4 \mathrm{~V}$ & $3 \mathrm{~V}$ & $\mathrm{~V}$ \\
\hline The Urine Clearance & $24 \mathrm{X}$ & $16 \mathrm{X}$ & $\mathrm{X}$ \\
\hline
\end{tabular}

A study published by Thornalley and colleagues appears to point a way forward.

\section{Summary of Thornalley's Work}

In table 1 is shown a summary of the essential data found in the "results and discussions" section of this study. It shows the behavior of thiamine in the blood plasma of diabetes patients and healthy subjects in a 24 -hour period.

The retention ratio $(15.85 / 64.1)$ of diabetic patients to healthy persons shows that polyuria reduces the water-soluble thiamine concentration in blood plasma of diabetes patients to $25 \%$ of the concentration required by normal healthy person to maintain 24 -hour vasodilatation in the microcirculation. It is assumed that all the watersoluble Vitamin B behaves in a similar manner.

If vitamin deficiency causes the development of diabetes complications, then all diabetes patients, including diabetes insipidus and diabetes mellitus patients, are susceptible to developing diabetes complications. Also, damage may be done in the organ rather than being caused by blockage of arteries leading to them.

Deficiency of water-soluble Vitamin B introduces significant change in the schematic representation in the definitions of diabetes and its progression as shown in flow-charts [2].

Even though Thornalley and colleagues did not use the term "polyuria" in the analysis of their work, the summary clearly shows the effect of polyuria on the concentration of thiamine in the blood plasma of diabetes patients. In fact, the same group subsequently conducted a clinical trial [5] to show the effect of thiamine on insipient kidney problems and interpreted their findings with the aid of the proteinglucose interaction (glycation) theory; completely ignoring the effect of polyuria as demonstrated in their previous work.

The data have been reinterpreted by the present author and used to explain the development of diabetes complications and and to propose their possible cure and prevention.

Diabetes complications are the long-term damage to organs with microcirculation, such as the brain and the nervous system, the eye, heart, kidney and the skin. Damage to the brain causes stroke, to the eye, blindness. The long-term damages include kidney and heart failures and amputations to lower limbs due to sores that do not heal.

The new interpretation of the findings has led to the proposition of a new theory to explain diabetes complications-"Vitamin B deficiency Theory" [6].

\section{New Theory}

It is assumed that the healthy persons do not develop tissue damage because the microcirculation is fully dilated for the full 24 hours and exchange of nutrients, gases and particles between blood and tissue is unhindered.

If the ratio (15.8/64.1) of retention of thiamine concentrations in the blood plasma of the diabetic patients to normal healthy persons at the end of 24 hours is applied to daily, diet-sourced, Vitamin B-including 


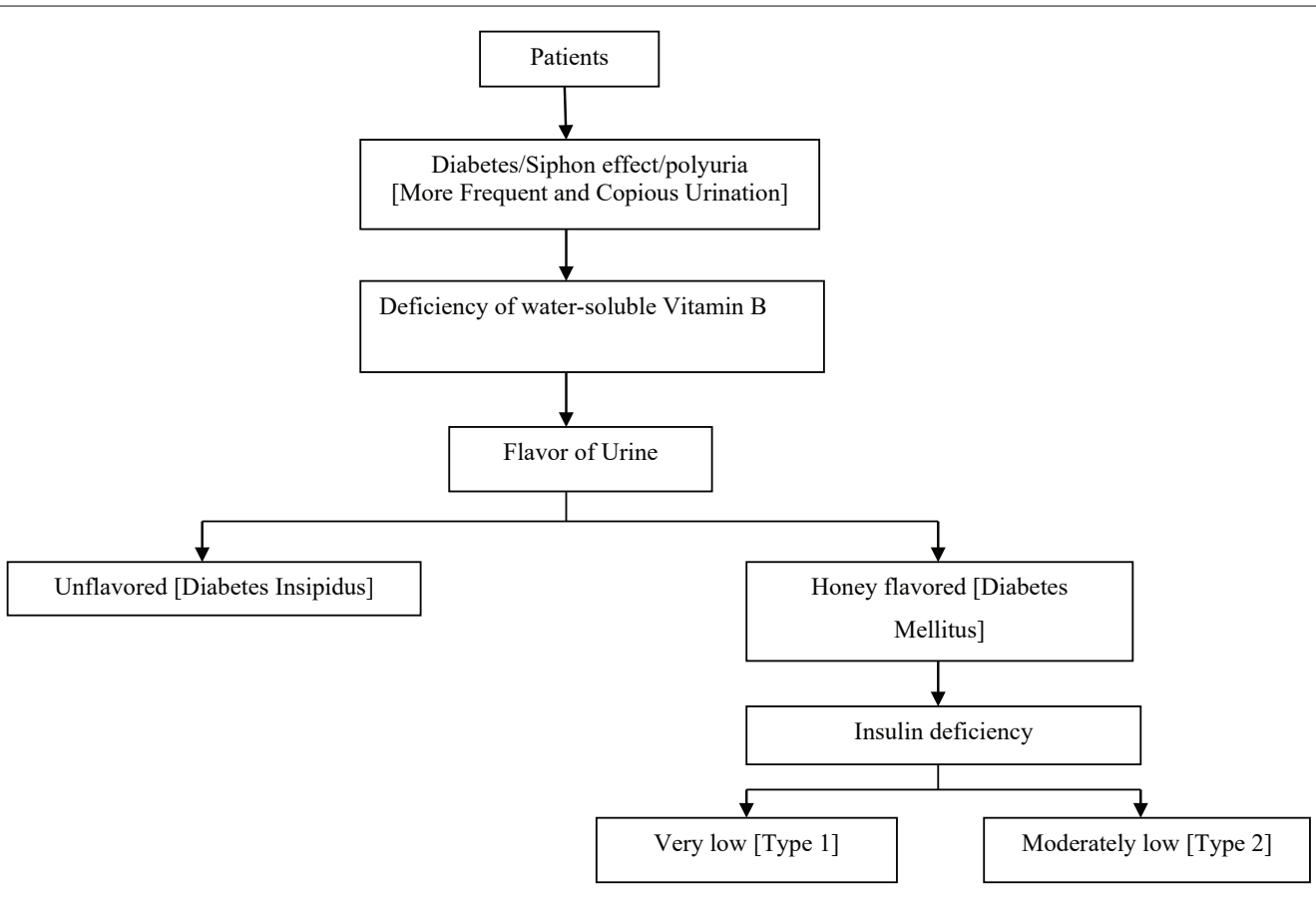

Flow-chart 2: Flow-chart of the definition of Diabetes.

vasodilators, niacin and thiamine-the following conclusions can be drawn.

- Polyuria reduces the total concentration of the vasodilators; thiamine and niacin, and the natural repair-kit-calcium-dpathothenate-to about $1 / 4$ the concentration needed by a healthy non-diabetic person to sustain full 24-hour vasodilatation in the microcirculation, and repair tissue damage.

- During the period of reduced concentration of the vasodilators, there is reduced blood supply in the microcirculation.

- Supply of blood, nutrients and oxygen to some cells are cut off in order to sustain energy production in cells.

- This results in daily tissue damage.

- Over time, (5-40 years), the cumulative tissue damage results in organ dysfunction-diabetes complications.

\section{Deductions from theory}

From the postulates of the theory, the following deductions can be made:

- Development of diabetes complications is independent of blood plasma glucose concentration.

- Diabetes Complications develop only in organs with microcirculation, e.g., the eye; kidney, heart, brain (nervous system) and skin.

- All complications in these organs are initiated simultaneously in the patient at the onset of diabetes, but symptoms of DC do not manifest at the same time in all susceptible organs.

- Any therapy that cures/prevents any manifested symptom in any one organ must cure/prevent manifested symptoms in all of the susceptible organs.

It follows that breaking or disrupting the chain of events outlined in the theory will stop or prevent tissue damage. Flowing from these deductions, the following hypothesis was enunciated.

\section{Hypothesis}

If a diabetes patient is given a daily supplementation of minimum concentration of vasodilators to sustain full 24-hour vasodilatation in the microcirculation, tissue damage will stop and damaged organs allowed healing naturally. This was tested.

\section{Experiment}

A tablet, Fenomin, containing nicotinamide, $15.0 \mathrm{mg}$; thiamine mononitrate, $12.5 \mathrm{mg}$; Calcium-d-pantothenate, $11.0 \mathrm{mg}$, was prepared and, in an open trial, given to patients to ingest one a day, after meals but before bed.

According to the theory tissue damage occurs daily, so there was no recommendation for end-time for patients to stop using the therapy. The patients used the tablets till they saw or felt improvement in their condition. The self-reports made to researchers over a seven-year period by over 200 people out of a total of about 950, are shown in table 2 .

The number 200 represents less than a $1 / 4$ of users of the therapy in the trial. It turned out that some people secured the tablets for relatives and friends and the researchers excluded the third party reports, however authentic and trustworthy they seemed. Others simply did not bother to report back.

They rejected reports, such as; "My mother was in hospital with chronic diabetic sore awaiting amputation. I heard of fenomin and bought two tubs for her. She was discharged from hospital and the sore is now completely healed. Thank you very much." And another, "my father is a prison warder who could not go to work because of a sore toe due to diabetes. I sent him some of your fenomin tablets and after six weeks he went back to work. Thank you for saving his job". 


\section{Results}

Table 2 shows some of the self-reports that the respondents gave. It shows methods of blood glucose control $v s$ type of diabetes complication cured/healed.

\section{Discussions}

Researchers face difficulty in doing a traditional placebo-controlled clinical trial because there are no easy methods of selecting suitable patients; for, there are no simple diagnostic tools to identify diabetes insipidus patients. The glucose theory simply ignores diabetes insipidus patients. The author chose what he has labelled, "open trial" method.

In this process, the patient identified the symptom that he/ she thought was caused by being a diabetic patient and, after using Fenomin tablets for sometime, reported the results.

Questions have been raised as to the appropriateness of this method of collecting data. It is claimed that it falls short of the gold standard of drug testing-"the randomized, placebo-controlled double blind clinical trial".

This usual method of drug testing is eminently suited to the situation when one seeks to transfer to the human situation, the results of laboratory studies, or animal experimental studies of, a new drug. Any cure is assumed to be a stochastic event and the results are statistically analysed. The study answers questions on efficacy, safety, possible side-effects, reaction with other drugs, etc.

The "open trial" study is different in its history, theory and objective. The therapy derives from in vivo experiment involving humans with substances familiar to the body-thiamine.

What was not known then was the physiological role these vitamins play in the body. So the trial was not to test for cure or healing but to validate the initial assumption that Vitamins B1 and B3 are the vasodilators that sustain the integrity of vasodilatation in the microcirculation for 24 hours.

The incurability of diabetes complications is well known. Curing of tissues already damaged by Calcium-D-pantothenate is also the natural function of the vitamin. That is why the need for a placebo control in the open trial was deemed otiose.

Vitamins B1, B3, and B5 are well characterised. At the levels used, the vitamins are safe. No side effects have also been found in the eight years the trial has been going. The trial is only about vasodilation in the microcirculation and not about safety and/or potential side-effects.

Diabetes complications have not been amenable to the traditional methods of treating diseases, so all the symptoms affecting all organs have not been systematically identified and chronicled. In such a case, it can only be assumed that the patient knows best.

This approach has been justified by the results. A list of diabetes complications has been compiled. Conditions such as; gum sores and unexpected falling down of diabetes patients, have been added to the list of symptoms that clinicians did not recognize as diabetes complications. Also, inability to have orgasm, inability to get erection, and the severe itching in the genital area, have all been found to be deteriorating sequences of the condition generally described as erectile dysfunction. Scratching of the itchy skin in the genital area tends to break the skin for sores to manifest. These are often fatal.

So the patients are best suited to observe their symptoms and report; no placebo is required. There is no reason to doubt the veracity of the reports. The results are their own validation; they do not require any statistical analysis for validation.

\section{Now, turning to the table of results}

Table 2 shows that the cure of early stage symptoms of diabetes complications by the therapy is independent of blood plasma glucose concentration. Also, the symptoms cured are found in all organs of the body. No conclusions could be drawn about damage to the brain and the heart as these organs do not show early symptoms of either stroke or heart failures. However, first time stroke patients who used fenomin reported quick and remarkable recovery.

The $2^{\text {nd }}$ column is the special case where the patients were supposedly, not even "diabetic" patients, but suffered symptoms similar to that of diabetic patients. When questioned, they all reported

Table 2: Shows some of the self-reports that the respondents gave. It shows methods of blood glucose control vs. type of diabetes complication cured/ healed.

\begin{tabular}{|l|c|c|c|c|c|c|}
\hline Method of blood control/ Complication type & Non diabetics & $\begin{array}{c}\text { T2 diet } \\
\text { control }\end{array}$ & T2 metformin & $\begin{array}{c}\text { T2 } \\
\text { Metformin+Gliclazide }\end{array}$ & $\begin{array}{c}\text { T2 } \\
\text { Metformin+Insulin }\end{array}$ & $\begin{array}{c}\text { T1 } \\
\text { insulin }\end{array}$ \\
\hline Swollen ankles/feet normalised & 3 & 5 & 8 & 11 & 3 \\
\hline Improved Visual acuity & 5 & 7 & 10 & 14 & 5 \\
\hline Feet/toes sore & 3 & 7 & 15 & 12 & 12 \\
\hline Gum sore & 1 & - & 2 & 2 & - \\
\hline Scalp sore & - & - & - & 1 & 1 & - \\
\hline Leg sore & 2 & 5 & 11 & 8 & 9 & 4 \\
\hline Warmed up Cold ands/feet & - & - & 13 & 7 & 2 \\
\hline Erectile function restored & 8 & 11 & 15 & 19 & 12 & 15 \\
\hline Severe itching in genital area stopped & 6 & - & 12 & 7 & 5 \\
\hline Semen production restored & - & - & 9 & 10 & 4 \\
\hline Numbness in toes/fingers disappeared & 9 & & 12 & 11 & - \\
\hline Burning foot syndrome & 15 & 12 & 21 & 23 & 7 \\
\hline
\end{tabular}


"urinating too much, including waking up more than two times in the night" and also that they came to know about Fenomin tablets through recommendation by friends who had been cured of symptoms similar to their own. It was suspected that these were Diabetes Insipidus patients-a condition not easily diagnosed by clinicians.

The "diet control" column shows that reducing blood sugar levels with "low carb diet" does not guarantee freedom from developing diabetes complications.

The theory recommends that patients continue to use the tablets as long as the diabetic condition remained, however, a large number of patients stopped using the tablets as soon as their ailing condition improved/cured. Some, who stopped for some time, came back on the tablets after 6-12 months when they felt recurrence of their symptoms or suspected new symptoms of diabetes complications affecting some other organ, manifested. This shows the inadequacy of "randomised placebo-controlled double-blind clinical trials" that have lasted for only a few months-usually, 3 months on drugs; and 2 months cooling off time $[7,8]$.

Some patients, including the author, have used the tablet regularly without break for the past 10 years and have not had any new symptoms or recurrence of the initial symptoms they had before using the tablets. They have not reported any side effects from the use of the tablets.

\section{Conclusion}

These results have validated the assumption that niacin and thiamine are the vasodilators that sustain the integrity of vasodilatation in the microcirculation. Also, a direct connection between polyuria and long-term chronic diabetes complications has been established. It has also been demonstrated that hypoglycaemia does not affect the development of diabetes complications; that Diabetes Insipidus patients also develop diabetes complications, thus making the current estimates of morbidities and mortalities due to diabetes very low. These estimates tend to exclude diabetes insipidus patients.

Above all, it has been demonstrated that diabetes complications are not a class of new diseases but manifestations of the damage of vitamin deficiency that can be cured and prevented by vitamin supplementation.

Apart from the cures, the fact that niacin and thiamine are the vasodilators that sustain the integrity of vasodilatation in the microcirculation, may have implications for diseases of the brain and the nerves, such as Parkinson's or Alzheimer's because it allows exchange of nutrients, gases and particles between blood and brain/ nerve tissues.

\section{Recommendation}

The polyuria theory has provided cure and prevention for diabetes complications, independently of hyperglycaemia. This may suggest that the cause of failure by the pancreas to produce sufficient insulin is different from the cause of polyuria. Consequently, the need is to solve two problems: the cause of polyuria and the cause of the failure by the pancreas to produce sufficient insulin all the time. It is believed that cure for diabetes can be found only through adopting this approach in research and clinical studies.

That the microcirculation is involved in organ damage has serious implications. Focused study on how microcirculation in a particular organ regulates the distribution of nutrients, gases, particles and drugs may well pave the way for finding treatment and cure for diseases such as hypertension, that affect organs with microcirculation, including the heart, kidney and the brain.

\section{Declaration}

This research has not received any funding from any source-public or private.

\section{References}

1. Oxford English Dictionary. Retrieved in 2011-09 23.

2. Thornalley PJ, Babaei-Jadidi R, Al Ali H, Rabbani N, Antonysunil A, et al. (2007) High prevalence of low plasma thiamine concentration in diabetes linked to a marker of vascular disease. Diabetologia 50: 2164-2170.

3. Rabbani N, Thornalley PJ (2011) Emerging role of thiamine therapy for prevention and treatment of early-stage diabetic nephropathy. Diabetes Obes Metab 13: 577-583.

4. Cousens G, Rainoshek D (2007) There is a Cure for Diabetes: The Tree of Life 21-Day+Program. North Atlantic Books, California 110.

5. Hammes HP, Du X, Edelstein D, Taguchi T, Matsumura T, et al. (2003) Benfotiamine blocks three major pathways of hyperglycaemic damage and prevents experimental diabetic retinopathy. Nat Med 9: 294-299.

6. Akyiaw BO (2015) Vitamin B Blood Plasma Deficiency Model for the Study of Diabetes Complications Demonstrates Potential for the Cure and Prevention of Complications in Type 2 Diabetes Mellitus Patients. J Diabetes Metab Disord Control 2: 49-52.

7. Rabbani N, Alam SS, Riaz S, Larkin JR, Akhtar MW, et al. (2009) High-dose thiamine therapy for patients with type 2 diabetes and microalbuminuria: a randomised, double-blind placebo-controlled pilot study. Diabetologia 52: 208-212.

8. Babaei-Jadidi R, Karachalias N, Ahmed N, Battah S, Thornalley PJ (2003) Prevention of incipient diabetic nephropathy by high-dose thiamine and benfotiamine. Diabetes 52: 2110-2120. 